\title{
ON IRREDUCIBLE CONTINUOUS CURVES
}

\author{
MARTIN G. ETTLINGER
}

This paper deals with the existence of continuous curves containing compact and closed point sets and with certain properties of continuous curves which are irreducible continua about point sets in spaces which are not necessarily metric. Previous results on these topics have been almost entirely for metric spaces. Thus Gehman ${ }^{1}$ proved for the plane that, given a compact continuum, there exists a compact continuous curve, which is the sum of a countable number of arcs plus its limit points, containing it. Whyburn and Ayres ${ }^{2}$ extended this to a space of a continuous curve in $n$ dimensions, and $\mathrm{Zippin}^{3}$ indicated that their argument might be modified to give the following: If $T$ is a closed and compact subset of a complete metric continuous curve $S$, there exists a compact continuous curve which is a subset of $S$ and contains $T$. Zippin ${ }^{4}$ proved that, given a complete metric continuous curve $S$ and a compact, closed, one-dimensional subset, $T$, of $S$, such that every component of $T$ is a continuous curve, and no more than a finite number of components of $T$ are of diameter greater than any positive number, there exists a compact continuous curve which is a subset of $S$ and an irreducible continuum about $T$. Miss Miller ${ }^{5}$ proved that if, in a connected space satisfying Axioms $0-2$ of R. L. Moore's Foundations of point set theory, ${ }^{6}{ }^{T}$ be a compact and closed point set, there exists a compact continuum containing $T$.

The concept of a continuum irreducible about a subset of itself was first introduced by Wilson. ${ }^{7}$ Most of the past work on continuous curves which are irreducible continua about point sets has been done

\footnotetext{
Presented to the Society, February 27, 1943; received by the editors January 21, 1943.

${ }^{1} \mathrm{H}$. M. Gehman, Concerning the subsets of a plane continuous curve, Ann. of Math. (2) vol. 27 (1926) p. 30.

${ }^{2}$ G. T. Whyburn and W. L. Ayres, On continuous curves in $n$ dimensions, Bull. Amer. Math Soc. vol 34 (1928) p. 350.

${ }^{3}$ L. Zippin, On continuous curves irreducible about subsets, Fund. Math. vol. 20 (1933) pp. 197-205.

${ }^{4}$ L. Zippin, loc. cit.

${ }^{5} \mathrm{H}$. C. Miller, $A$ theorem concerning closed and compact point sets which lie in connected domains, Bull. Amer. Math. Soc. vol. 46 (1940) p. 848.

${ }^{6}$ R. L. Moore, Foundations of point set theory, Amer. Math. Soc. Colloquium Publications vol. 13, New York, 1932.

${ }^{7} \mathrm{~W}$. A. Wilson, On the oscillation of a continuum at a point, Trans. Amer. Math. Soc. vol. 27 (1925) p. 433.
} 
by Gehman, who obtained results ${ }^{8}$ equivalent to the following theorem for the plane: If $M$ be a compact irreducible continuous curve about a closed point set $K$, then (1) $M$ is an irreducible continuum about $K$; (2) $K$ contains all the non-cut points of $M$; (3) if $H$ is a subcontinuum of $M, H$ is connected im kleinem at every point of $H(M-K) . \mathrm{He}^{9}$ gave a later proof of part (2) which with very slight modification shows that if, in a space satisfying Axioms $0-1, M$ be a locally compact continuous curve which is an irreducible continuum about a closed subset $K$ of $M, K$ contains all the non-cut points of $M$. Zippin ${ }^{10}$ indicated a proof of part (1) if $M$ be a complete metric space and $K$ be compact.

TheOREM 1. In a connected space satisfying Axioms 0-2, every closed and compact point set $T$ which has no continuum of condensation is a subset of a compact hereditary continuous curve.

Proof. Every component of $T$ is a continuous curve and hence is arcwise connected. By Axiom 2 regions may be considered as connected domains. There exists ${ }^{11}$ an infinite sequence $W_{1}, W_{2}, W_{3}, \cdots$ such that (1) for each $n W_{n}$ is a finite subcollection of $G_{n}$ covering $T$; (2) if $g$ be a member of $W_{n+1}, \bar{g}$ is a subset of some member of $W_{n}$; (3) if $H$ and $K$ be two mutually exclusive closed subsets of $T$, and $k$ be a positive integer, there exists a positive integer $m$ such that if $U$ be a coherent collection of $k$ regions, each belonging to a member of the sequence $W_{m}, W_{m+1}, \cdots$, and $U^{*}$ contain a point of $H,{ }^{12}$ then $U^{*}$ contains no point of $K$; (4) every region of each $W_{n}$ contains a point of $T$, and if $P_{1}, P_{2}, \cdots$ be a sequence of points such that for each $n P_{n}$ is a subset of $W_{n}$, then some subsequence of that sequence converges to a point of $T$. For each region $R_{i_{1}}$ in $W_{1}$ let $P_{i_{1}}$ be a point of $R_{i_{1}} \cdot T$. Let $\alpha_{i_{1}}$ denote an arc from $P_{1_{1}}$ to $P_{i_{1}}$ which is constructed to lie in the component of $T$ containing $P_{1_{1}}$ if that component contains $P_{i_{1}}$. Let $\alpha_{1}$ be the sum of all $\alpha_{i_{1}}$. For each pair of regions, $R_{i_{1}}$ of $W_{1}$ and $R_{i_{2}}$ of $W_{2}$, such that $R_{i_{1}}$ contains $R_{i_{2}}$, let $P_{i_{1} i_{2}}$ be a point of $R_{i_{2}} \cdot T$. Let $\alpha_{i_{1} i_{2}}$ denote an arc from $P_{i_{1}}$ to $P_{i_{1} i_{2}}$ lying in $R_{i_{1}}$ or in the component of $T$ which contains $P_{i_{1}}$ according as that component does not or does contain $P_{i_{1} i_{2}}$. Let $\alpha_{2}$ be the sum of $\alpha_{1}$ and all $\alpha_{i_{1} i_{2}}$. For each

${ }^{8}$ H. M. Gehman, Irreducible continuous curves, Amer. J. Math. vol. 49 (1927) pp. $189-196$.

$\because \mathrm{H}$. M. Gehman, Concerning certain types of non-cut points, with an application to continuous curves, and Concerning irreducible continua, Proc. Nat. Acad. Sci. U.S.A. vol. 14 (1928) pp. 431-435.

${ }^{10} \mathrm{~L}$. Zippin, loc. cit.

11 R. L. Moore, loc. cit. pp. 58-59, Theorem 81.

12 The point set $U^{*}$ is the sum of all point sets of the collection $U$. 
triplet of regions, $R_{i_{1}}$ of $W_{1}, R_{i_{2}}$ of $W_{2}$, and $R_{i_{3}}$ of $W_{3}$, such that $R_{i_{1}}$ contains $R_{i_{2}}$ which contains $R_{i_{3}}$, let $P_{i_{1} i_{2} i_{3}}$ be a point of $R_{i_{3}} \cdot T$. Let $\alpha_{i_{1} i_{2} i_{3}}$ denote an arc from $P_{i_{1} i_{2}}$ to $P_{i_{1} i_{2} i_{3}}$ lying in $R_{i_{2}}$ or in the component of $T$ which contains $P_{i_{1} i_{2}}$ according as that component does not or does contain $P_{i_{1} i_{2} i_{3}}$. Let $\alpha_{3}$ be the sum of $\alpha_{2}$ and all $\alpha_{i_{1} i_{2} i_{3}}$. Continue the indicated constructions indefinitely. Let $\alpha$ be the sum of all $\alpha_{n}$.

The point set $\bar{\alpha}$ is a hereditary continuous curve containing $T$. First, since $\alpha$ is the sum of a monotonic sequence of connected sets, it is connected, so that $\bar{\alpha}$ is a continuum. Second, $\bar{\alpha}$ contains $T$ because, given a point $P$ of $T$ and a region $R$ containing $P$, there exists for some $n$ a region $R_{i_{n}}$ of $W_{n}$ which is a subset of $R$ and contains $P$; there then exists a monotonic sequence of regions $R_{i_{1}}$, $R_{i_{2}}, \cdots, R_{i_{n}}$, so that $R_{i_{n}}$ contains a point $P_{i_{1}} \ldots i_{n}$ of $\alpha$; whence $R$ contains a point of $\alpha$ and $P$ is a limit point of $\alpha$.

Suppose that $\alpha$ contains an infinite point set $K$ such that $\alpha+T$ contains no limit point of $K$. For each $n, \alpha_{n}+T$ is compact and therefore contains only a finite number of points of $K$. Since $W_{n}$ covers $\alpha-\left(\alpha_{n}+T\right), W_{n}^{*} \cdot K$ is infinite. This contradicts part (4) of the condition on the $W_{n}$ 's. It follows that $\bar{\alpha}$ is compact and that $T$ contains $\bar{\alpha}-\alpha$.

It remains to prove that every subcontinuum of $\bar{\alpha}$ is a continuous curve. Suppose that this is not true. Then there exists a subcontinuum $M$ of $\bar{\alpha}$, containing a point $P$ at which it is not connected im kleinem. Suppose, first, that $P$ is not a point of $T$. By part (4) of the condition on the $W_{n}$ 's there exists an integer $k$ such that no region $W_{k-1}$ contains $P$; by part (2), if $R_{i_{k}}$ is a region of $W_{k}, \bar{R}_{i_{k}}$ does not contain $P$. As $W_{k}$ is a finite collection there exists a region $R$ containing $P$ that contains no point of $W_{k}^{*}$ and so contains no point of $T$. Hence $R \cdot \bar{\alpha}$ is a subset of $R \cdot \alpha_{k}$. As $R$ contains the point $P$ at which $M$ is not connected im kleinem, it contains a continuum of condensation of $M$ and so of $\alpha_{k}$, the sum of a finite number of arcs. This is impossible.

Suppose now that $P$ is a point of $T$. There exist a domain $D$ containing $P$, and a sequence of mutually exclusive continua $K, M_{1}$, $M_{2}, \cdots$ converging to $K$, such that (1) $K$ contains $P$; (2) every continuum of the sequence contains a point of $D$ and a point of $\bar{D}-D$; (3) each $M_{j}$ is a component of $M \cdot \bar{D}$. Since it contains no point where $M$ is connected im kleinem, $K \cdot D$ is a subset of $T$. If every point of $K \cdot D$ is a limit point of $T-K \cdot D$, then if $D^{\prime}$ be a domain intersecting $K, \bar{D}^{\prime}$ a subset of $D$, some component of $K \cdot \bar{D}^{\prime}$ will be nondegenerate and a continuum of condensation of $T$. Hence 
some point $P^{\prime}$ of $K \cdot D$ is not a limit point of $T-K \cdot D$. Let $D^{\prime}$ be a domain containing $P^{\prime}$, such that $\bar{D}^{\prime}$ is a subset of $D$ and contains no point of $T-K \cdot D$. Let $D^{\prime \prime}$ be a domain containing $P^{\prime}$ such that $\bar{D}^{\prime \prime}$ is a subset of $D^{\prime}$. Let $D^{\prime \prime \prime}$ be a domain containing $P^{\prime}$ such that $\bar{D}^{\prime \prime \prime}$ is a subset of $D^{\prime \prime}$. Let $K^{\prime}$ be the component of $K \cdot \bar{D}^{\prime \prime \prime}$ containing $P^{\prime}$. $K^{\prime}$ is a nondegenerate subcontinuum of $T$ and a subset of the limiting set of the sequence $M_{1} \cdot \bar{D}^{\prime \prime}, M_{2} \cdot \bar{D}^{\prime \prime}, \ldots$ By parts (3) and (4) of the conditions on the $W_{n}$ 's, there exists an integer $k$ such that no region of $W_{n}, n \geqq k$, intersects $\bar{D}^{\prime \prime}$ and $S-D^{\prime} . \alpha_{i_{1}} \cdots i_{n}$ lies in $R_{i_{n-1}}+T$, so every arc $\alpha_{i_{1}} \ldots i_{n}, n>k$, which intersects $\bar{D}^{\prime \prime}$ and $S-D^{\prime}$ lies in $T$. Hence every arc $\alpha_{i_{1}} \cdots i_{n}, n>k$, which intersects $\bar{D}^{\prime \prime}$ lies in $D^{\prime}$ or in $T$; both end points of such an arc lie in $T$, by its construction, so that either the whole arc lies in $T$ or its end points lie in $T \cdot D^{\prime}$ and so in $K$; in the latter case the end points lie in the same component of $T$ and so the arc lies in $T$. Therefore $\bar{\alpha} \cdot D^{\prime \prime}$ is a subset of $\alpha_{k}+T$.

It follows that $M_{1} \cdot \bar{D}^{\prime \prime}, M_{2} \cdot \bar{D}^{\prime \prime}, \cdots$ are all subsets of $\alpha_{k}+T$. Therefore $K^{\prime}$ is a continuum of condensation of $\alpha_{k}+T . K^{\prime} \cdot\left(\overline{T-K^{\prime}}\right)$ and $K^{\prime} \cdot\left(\overline{\alpha_{k}-\alpha_{k} \cdot K^{\prime}}\right)$ are closed and totally disconnected. But their sum is the continuum $K^{\prime}$. This involves a contradiction.

ThEOREM 2. In a connected space satisfying Axioms 0-2, every closed and compact point set $T$ is a subset of a compact continuous curve.

Proof. By Axiom 2 regions may be considered as connected domains. Construct the $W_{n}$ 's and the $P_{i_{1}} \ldots i_{n}$ 's exactly as in the proof of Theorem 1. Define the $\alpha_{i_{1}} \ldots i_{n}$ 's as follows: $\alpha_{i_{1}}$ is an arc from $P_{1_{1}}$ to $P_{i_{1}} ; \alpha_{i_{1}} \ldots i_{n}$ is an arc from $P_{i_{1} \ldots i_{n-1}}$ to $P_{i_{1}} \ldots i_{n}$ in $R_{i_{n-1}}$. Define $\alpha_{n}$ and $\alpha$ as before on this basis. It will be proved that $\bar{\alpha}$ is a compact continuous curve containing $T$.

By the argument used in the proof of Theorem 1 it may be shown that $\bar{\alpha}$ is a compact continuum containing $T$. It remains to show that $\bar{\alpha}$ is connected im kleinem at every point. Let $P$ be any point of $\bar{\alpha}$, and $R$ a region containing $P$. There exists a positive integer $k$ such that if $R_{1}$ and $R_{2}$ are any two intersecting regions of $W_{k}$, one containing $P$, then $R$ contains $\bar{R}_{1}+\bar{R}_{2}$. For each set of indices $i_{1}, i_{2}, \cdots, i_{k}$ let $L_{i_{1}} \ldots i_{k}$ be the sum of all $\alpha_{i_{1}} \ldots i_{n}$ 's, $n>k$, whose first $k$ indices are the members of that set. The set of $L_{i_{1}} \ldots i_{k}$ 's is finite. If $\alpha_{k}$, the sum of a finite number of arcs, contains $P$, it is connected im kleinem there. Let $R^{\prime}$ be a region containing $P$ such that (1) if $P^{\prime}$ is a point of $\alpha_{k} \cdot R^{\prime}$, there is a connected subset of $\alpha_{k} \cdot R$ containing $P$ and $P^{\prime}$; (2) if $R^{\prime}$ contains a point of $\overline{L_{i_{1} \cdots i_{k}}}, \overline{L_{i_{1}} \cdots i_{k}}$ contains $P$. Let $Q$ be any 
point of $R^{\prime} \cdot \bar{\alpha}$. If $Q$ is a point of $\alpha_{k}$, there is a connected subset of $R \cdot \bar{\alpha}$ containing $P$ and $Q$. Suppose it does not belong to $\alpha_{k}$. Then it must belong to some $L_{i_{1}} \cdots i_{k}$. Then that $L_{i_{1} \cdots i_{k}}$ contains $P$ and is a subset of $\bar{R}_{i_{k}}$. Since $\bar{R}_{i_{k}}$ contains $P$ it is a subset of $R$. Hence $L_{i_{1}} \cdots i_{k}$ is a subset of $R \cdot \bar{\alpha}$ which is plainly connected and contains $P$ and $Q$. The theorem is therefore proved.

Theorem 2 does not remain true if "locally compact" replaces "compact." For let space be the sum of the intervals in the plane from $(0,0)$ to $(1,0)$ and from $(1 / n, 0)$ to $(1 / n, 1),(n>0)$, and let $T$ be the set of points $(1 / n, 1)$. Here space is a locally non-compact continuous curve which is an irreducible continuum about $T$. Furthermore, there is a non-cut point of space not belonging to $T$.

'Theorem 3. If space satisfies Axioms $0-1$, and $M$ is an irreducible continuous curve about a compact and closed subset $T$ of $M, M$ is a compact irreducible continuum about $T$.

Proof. By Theorem 2, $M$ contains a compact continuous curve $U$ containing $T$. Since it is identical with $U, M$ is compact.

Suppose that there exists a proper subcontinuum $V$ of $M$ containing $T$. Let $P$ be a point of $M-V$, and let $C$ be the component of $M-P$ containing $V$. The set $C$ is a domain with respect to $M$ and hence considered as space satisfies Axioms $0-2$. Hence, by Theorem 2, $C$ contains a compact continuous curve $U^{\prime}$ containing $T$. This is a contradiction.

TheOREM 4. If space satisfies Axioms $0-1$, and $M$ is a locally compact continuous curve which is an irreducible continuum about a closed subset $T$ of $M$, every continuum of condensation of $M$ is a continuum of condensation of $T$.

Proof. The following lemma must first be demonstrated:

LEMma. Under the conditions of Theorem 4, every continuum of condensation of $M$ is a subset of $T$.

Proof of lemma. Regard $M$ as space. Then Axiom 2 holds. Suppose that $M$ has a continuum of condensation $N$ which does not lie wholly in $T$. Since $M$ is locally compact, $N$ contains a nondegenerate compact continuum $N^{\prime}$ which lies in $M-T . N^{\prime}$ contains a subcontinuum $N^{\prime \prime}$ which is an irreducible continuum between two points $A$ and $B$. Every component of $M-N^{\prime \prime}$ intersects $T$ since its complement is a continuum. Since $M$ is locally compact, $N^{\prime \prime}$ is a subset of a domain $D$ such that $\bar{D}$ is a compact subset of $M-T$. The set $\bar{D}-D$ is the sum of its intersections with the components of $M-N^{\prime \prime}$, and 
each component intersects it since the component has a limit point in $N^{\prime \prime}$ and in $T$. Since no closed and compact point set is the sum of infinitely many mutually exclusive open subsets of itself, $M-N^{\prime \prime}$ has only a finite number of components. As every point of $N^{\prime \prime}$ is a cut point of $M,{ }^{13}$ every non-cut point of $N^{\prime \prime}$ is the boundary of some component of $M-N^{\prime \prime}$, so the set of all non-cut points of $N^{\prime \prime}$ is finite. There exists a component $K$ of $M-N^{\prime \prime}$ such that $\bar{K} \cdot N^{\prime \prime}$ is infinite. There then exist three points of $\bar{K} \cdot N^{\prime \prime}$ which lie on the segment $A B$ of $N^{\prime \prime}$, such that no one of them is the entire boundary of any component of $M-N^{\prime \prime}$. Let $P$ be that one of them which lies between the other two on the segment $A B$ of $N^{\prime \prime} .^{14}$ Then $K+N^{\prime \prime}-P$ is plainly connected. Since $N^{\prime \prime}-P$ contains a limit point of every component of $M-N^{\prime \prime}, M-P$ is connected, which leads to a contradiction.

Suppose now that Theorem 4 is not true. Then, by the Lemma, $T$ contains a continuum $N$ which is a continuum of condensation of $M$ but not of $T$. Let $P$ be a point of $N$ not belonging to $\overline{T-N}$. Let $Q$ be a point of $N-P$. Let $C$ be any subcontinuum of $M$ which contains $\overline{T-N}+Q$. Since it contains $T$, the continuum $C+N$ is $M$. Hence $C$ contains $M-N$, and therefore $N$, so that $C$ is $M$ and $M$ is an irreducible continuum about $\overline{T-N}+Q$. As $N$ contains a point $P$ not in $\overline{T-N}+Q$, this contradicts the Lemma, and the theorem is proved.

Theorem 5. In a connected space satisfying Axioms $0-2$, every compact and closed point set $T$ with no continuum of condensation is a subset of a compact continuous curve which has no continuum of condensation.

Proof. By Theorem 1 there exists a compact hereditary continuous curve $K$ containing $T$. The set $K$ contains a continuum $M$ which is an irreducible continuum about $T$. Since $M$ is a continuous curve, by Theorem $4 M$ has no continuum of condensation.

In conclusion I wish to express my deepest thanks to Professor R. L. Moore for invaluable guidance in the preparation of this paper.

The University of TeXas

${ }^{13}$ H. M. Gehman, loc. cit.

${ }^{14}$ R. L. Moore, loc cit. pp. $35 \mathrm{ff}$. 